\title{
Desarrollos relevantes en Evaluación Psicológica y Psicometría: Editorial
}

\section{Relevant developments in Psychological Assessment and Psychometrics: Editorial}

\author{
Karina Rdz-Navarro ${ }^{\mathrm{a}}$ Eugenia V. Vinet ${ }^{\mathrm{b}}$ \\ Editoras invitadas \\ ${ }^{a}$ Universidad Complutense de Madrid, Madrid, España ${ }^{b}$ Universidad de La Frontera, Temuco, Chile
}

Desde sus inicios la Psicología ha estado íntimamente ligada al desarrollo de métodos y técnicas de investigación y, debido a su utilidad, muchos de ellos han traspasado las fronteras de la disciplina, convirtiéndose en un importante aporte para otros campos de la ciencia.

Por ejemplo, Thurstone, Likert, Guttman y Osgood, desarrollaron procedimientos de construcción de instrumentos de medición que son utilizados frecuentemente en investigación en Psicología, Sociología, Educación, Marketing, Economía, Ciencias de la Salud, entre otras. Campbell, Cook y Stanley contribuyeron al desarrollo y difusión de los diseños experimentales; Glass y Cohen hicieron importantes aportes a la creación del Metaanálisis; y Moreno fundó la Sociometría y lo que hoy conocemos como Análisis de Redes.

A su vez, desde la Psicología se han desarrollado importantes aportes a la Estadística, entre los cuales destacan, por ejemplo, los trabajos de: Galton en correlación y regresión; Baron y Kenny en análisis de mediación y moderación estadística; Spearman y Thurtone en Análisis Factorial; Cronbach en fiabilidad y Teoría de la Generalizabilidad; Lord, Novic, Rasch, Birbaum y Samejima en el desarrollo de modelos de Teoría de Respuesta a los Ítems (TRI), solo por mencionar algunos.

La Metodología en general, y la Estadística y la Psicometría en particular, avanzan de manera acelerada, sin embargo, estos avances tardan en materializarse en los estudios aplicados, pues su masificación depende tanto de su difusión entre los investigadores, como de los desarrollos informáticos que permitan facilitar su uso e implementación. Afortunadamente, los avances en el desarrollo de software adaptados a los usuarios (e.g., SPSS, LISREL, MPlus, R, STATA, etc.) han facilitado el acceso a las innovaciones metodológicas recientes, sin embargo, la difusión de estos avances entre los investigadores aplicados es todavía escasa.

En este contexto, al ser invitadas como Editoras de esta sección temática hemos querido contribuir a la difusión de algunos avances metodológicos en el área de la Psicometría y la Evaluación Psicológica, invitando a investigadores nacionales y extranjeros que trabajan en estos campos de la Psicología. El éxito de la convocatoria y la calidad de los trabajos recepcionados superaron nuestras expectativas, dando pie para que podamos plantear dos secciones monográficas consecutivas (volumen 23, números 1 y 2).

Cómo citar:

Rdz-Navarro, K. y Vinet, E. V. (2014). Desarrollos relevantes en evaluación psicológica y psicometría: Editorial. Revista de Psicología, 23(1), 1-2. doi: $10.5354 / 0719-0581.2014 .32880$ 
Para este primer número hemos seleccionado seis artículos con fuerte contenido metodológico aplicado a la construcción y validación de instrumentos de medida de interés para la Psicología; los cuales exploran la aplicación de una amplia gama de métodos: Teoría Clásica de los Tests (TCT), Teoría de la Generalizabilidad (TG), Teoría de Respuesta al Ítem (TRI), Análisis Factorial Exploratorio (AFE) y Confirmatorio (AFC) y Ecuaciones Estructurales (SEM).

Así, Orozco-Parra y Domínguez-Espinosa, nos presentan la Escala de Actitud Religiosa para creyentes en religiones teístas, construida utilizando los principios de la TCT y validada utilizando AFE y AFC.

Retomando los principios de la TG, Gempp revisa la utilidad del estadístico Phi(Lambda) para estimar la fiabilidad de una medida en un punto de corte dado, aplicándolo a la selección de personal, y presenta un software de distribución gratuita, diseñado por el propio autor, que permite estimar dicho coeficiente.

Utilizando los principios del AFC, Caneto, Cupani y Pilatti comparan tres modelos métricos distintos para evaluar las propiedades psicométricas del Cuestionario de Motivos de Consumo de Alcohol.

En una línea similar, Reyna, Ortiz y Revilla comparan la técnica de AFC con los procedimientos modernos de Ecuaciones Estructurales Exploratorias (E-SEM) para analizar las respuestas de los sujetos a un test sobre estilos de decisión.

Por su parte, Junco y Prieto nos muestran una interesante aplicación del Modelo de Rasch a la validación de un test neuropsicológico para la evaluación de demencia.

Finalmente, Revuelta y Ximénez revisan los desarrollos más actuales de la TRI con procedimientos bayesianos multinivel y muestran sus potencialidades para el análisis de instrumentos de medida aplicados a poblaciones con distintos niveles de agregación, presentando a su vez, un código que permite estimar dichos modelos con el software OpenBugs.

Esperamos que, a través de estos artículos, los lectores de la Revista de Psicología puedan formarse una opinión no solo de los fundamentos de cada una de las técnicas utilizadas, sino también de las potencialidades y ventajas que ofrecen los métodos clásicos y modernos de análisis al ser aplicados a la Psicometría y la Evaluación Psicológica. 